\title{
Risk of venous thromboembolism in women with polycystic ovary syndrome: a population-based matched cohort analysis
}

\author{
Steven T. Bird PharmD MS, Abraham G. Hartzema PhD PharmD, James M. Brophy PhD MD, \\ Mahyar Etminan PharmD MS, Joseph A.C. Delaney PhD
}

\begin{abstract}
- Abstract
Background: There is an increased risk of venous thromboembolism among women taking oral contraceptives. However, whether there is an additional risk among women with polycystic ovary syndrome (PCOS) is unknown.

Methods: We developed a population-based cohort from the IMS LifeLink Health Plan Claims Database, which includes managed care organizations in the United States. Women aged 1846 years taking combined oral contraceptives and who had a claim for PCOS $(n=43506)$ were matched, based on a propensity score, to control women ( $n=43506)$ taking oral contraceptives. Venous thromboembolism was defined using administrative coding and use of anticoagulation. We used Cox proportional hazards models to assess the relative risk (RR) of venous thromboembolism among users of combined oral contraceptives with and without PCOS.

Results: The incidence of venous thromboembolism among women with PCOS was 23.7/ 10000 person-years, while that for matched

controls was 10.9/10 000 person-years. Women with PCOS taking combined oral contraceptives had an RR for venous thromboembolism of 2.14 (95\% confidence interval $[\mathrm{Cl}]$ 1.41-3.24) compared with other contraceptive users. The incidence of venous thromboembolism was 6.3/10 000 person-years among women with PCOS not taking oral contraceptives; the incidence was 4.1/10 000 personyears among matched controls. The RR of venous thromboembolism among women with PCOS not taking oral contraceptives was 1.55 (95\% Cl 1.10-2.19).

Interpretation: We found a 2-fold increased risk of venous thromboembolism among women with PCOS who were taking combined oral contraceptives and a 1.5-fold increased risk among women with PCOS not taking oral contraceptives. Physicians should consider the increased risk of venous thromboembolism when prescribing contraceptive therapy to women with PCOS.
\end{abstract}

$\mathrm{P}$ olycystic ovary syndrome (PCOS) is the most common endocrine disorder among women of reproductive age. The National Institutes of Health criteria estimates its prevalence in the United States to be between $6 \%$ and $10 \%$, while the Rotterdam criteria estimates the prevalence to be as high as $15 \% .^{1}$ Although its cause is not entirely known, the diagnostic criteria include oligo- or anovulation, clinical and/or biochemical signs of hyperandrogenism, and polycystic ovaries. ${ }^{2}$ Women often present with clinical manifestations of high androgen levels, including facial hair growth (hirsutism), acne vulgaris and hair loss on the scalp. Previous studies reported the prevalence of impaired glucose tolerance to be $31.1 \%-35.2 \%$ and the prevalence of type 2 diabetes to be $7.5 \%-9.8 \%$ among women with PCOS. ${ }^{3,4} \mathrm{~A}$ recent consensus workshop reported that the prevalence of several known risk factors for cardiovascular disease (hypertension, diabetes, abdominal obesity, psychological factors, smoking, altered apoA1/
ApoB ratios) are doubled among women with PCOS compared with matched controls. ${ }^{1,5}$

Combined oral contraceptives are the mainstay treatment for PCOS. However, they are also known to elevate the risk of venous thromboembolism and cardiovascular disease. ${ }^{6}$ To date, contraceptive studies involving women with PCOS have focused mainly on efficacy, evaluating the effect of combined oral contraceptives on the reduction of hirsutism and hyperandrogenism. ${ }^{7.8}$ Two studies assessed the metabolic effects of combined oral contraceptives in PCOS, but these studies had small sample sizes and could not evaluate for cardiovascular events., ${ }^{9}$

Although women with PCOS have an increase in both cardiovascular risk factors and subclinical cardiovascular disease,,$^{11}$ recent guidelines have concluded there are no data in the literature assessing the association between the use of oral contraceptives and cardiovascular disease among women with PCOS. ${ }^{2}$ Because combined oral contraceptives are the mainstay
Competing interests: Abraham Hartzema has served as a consultant for Pfizer and the Observational Medical Outcomes Partnership. None declared by all other authors.

This article has been peer reviewed.

Correspondence to:

Steven Bird, bird.steven@gmail.com

CMAJ 2013. DOI:10.1503 /cmaj.120677 
treatment, our objective was to determine whether women with PCOS taking combined oral contraceptives have a greater risk of venous thromboembolism compared with other contra-

Table 1: Health care and combined oral contraceptive use at baseline

\begin{tabular}{|lcc|}
\hline Characteristic & $\begin{array}{c}\text { Polycystic ovary } \\
\text { syndrome* }\end{array}$ & $\begin{array}{c}\text { Matched } \\
\text { controls }\end{array}$ \\
\hline Women, no. & 43506 & 43506 \\
\hline Age, yr, mean & 28.7 & 28.9 \\
\hline Health care use, \% of women & 3.57 & 4.05 \\
\hline Admission to hospital & 4.79 & 4.45 \\
\hline Emergency department visit & 51.31 & 50.08 \\
\hline $\begin{array}{l}\text { Physician office visit } \\
\text { Combined oral contraceptive use }\end{array}$ & $20150(46.32)$ & $20278(46.61)$ \\
\hline $\begin{array}{l}\text { New user, } \dagger \text { no. (\%) of women } \\
\text { Prevalent user, } \neq \text { no. (\%) of women }\end{array}$ & $2356(53.68)$ & $23228(53.39)$ \\
\hline $\begin{array}{l}\text { Prior time on combined oral } \\
\text { contraceptives, mean, } d\end{array}$ & 103 & 103 \\
\hline $\begin{array}{l}\text { Total time on combined oral } \\
\text { contraceptives, mean, } d\end{array}$ & 201 & 202 \\
\hline $\begin{array}{l}\text { Number of combined oral } \\
\text { contraceptives products used in the } \\
\text { past, mean }\end{array}$ & 0.6 & 0.6 \\
\hline
\end{tabular}

\section{Combined oral contraceptive, $\%$ of women}

Desogestrel, $\mu \mathrm{g}$ ethinyl estradiol

\begin{tabular}{|crr|}
\hline 20 & 3.45 & 3.38 \\
\hline 25 & 0.47 & 0.46 \\
\hline 30 & 8.39 & 8.31 \\
\hline Drospirenone, $\mu$ g ethinyl estradiol & & \\
\hline 20 & 7.46 & 8.45 \\
\hline 30 & 20.81 & 21.02 \\
\hline
\end{tabular}

Levonorgestrel, $\mu$ g ethinyl estradiol

\begin{tabular}{|lll|}
\hline 20 & 5.19 & 5.14 \\
\hline 30 & 3.75 & 3.72 \\
\hline Triphasic & 2.35 & 2.11 \\
\hline Norethindrone, $\mu$ g ethinyl estradiol & & \\
\hline
\end{tabular}

$\begin{array}{lll}35 & 10.53 & 10.28\end{array}$

Norethindrone acetate, $\mu \mathrm{g}$ ethinyl estradiol

\begin{tabular}{|lll|}
\hline 20 & 4.60 & 4.48 \\
\hline 30 & 2.74 & 2.66 \\
\hline 35 & 1.51 & 1.55 \\
\hline
\end{tabular}

Norgestimate, ethinyl-estradiol $\mu \mathrm{g}$

\begin{tabular}{|crc|}
\hline 25 & 5.78 & 5.68 \\
\hline 35 & 18.31 & 18.21 \\
\hline Norgestrel, $\mu$ g ethinyl estradiol & & \\
\hline 30 & 4.66 & 4.55 \\
\hline
\end{tabular}

*Defined as a claim for polycystic ovary syndrome (International Classification of Disease, 9th Revision [Clinical Modification (ICD-9-CM)] 256.4).

tInitiated combined oral contraceptive therapy after the polycystic ovary syndrome claim.

\#Evidence of combined oral contraceptive use during the 365 -day period before the polycystic ovary syndrome claim. ceptive users. We also examined whether women with PCOS not taking oral contraceptives had an increased risk of venous thromboembolism compared with the general population.

\section{Materials and methods}

\section{Data source}

The IMS LifeLink Health Plan Claims Database contains paid claims data from over 102 managed care plans in the US. This database contains fully adjudicated medical and pharmacy claims for over 68 million patients, including inpatient and outpatient diagnoses and procedures (International Classification of Diseases, 9th Revision [Clinical Modification (ICD-9-CM)] in addition to retail and mail-order prescription records. The data are considered to be reasonably representative of US residents with private health insurance in terms of geography, age and sex, and these data have been previously used to evaluate the comparative safety of combined oral contraceptives. ${ }^{12-14}$ The LifeLink database is subject to quality checks to ensure data quality and to minimize error rates. ${ }^{15}$

This study was approved by the University of Florida Gainsville Health Science Institutional Review Board.

\section{Study population}

The study period was May 1, 2001, to Dec. 31, 2009. Women between the ages of 18 and 46 years were included in the analysis. The inception cohort was based on exposure to one of the following combined oral contraceptives containing $\leq 0.035 \mathrm{mg}$ ethinyl estradiol: desogestrel, drospirenone, levonorgestrel, norethindrone, norethindrone acetate, norgestimate or norgestrel.

Because we required a 1-year period for baseline covariate assessment, we excluded women who did not have 1 year of total enrolment in the IMS database. We also excluded women with a history of cancer, cerebrovascular disease, cardiovascular disease, venous thromboembolism or prior anticoagulation (warfarin and heparin). Censoring occurred at the outcome of venous thromboembolism, after a gap in combined oral contraceptive therapy of 30 or more days, discontinuation of enrolment, and the end of the study period. Because venous thromboembolism risk varies substantially between person-time exposed to and unexposed to combined oral contraceptives, the exposure cohort was limited strictly to exposed person-years to increase internal validity of the study and to reduce concern for confounding by contraceptive use.

For inclusion in the PCOS cohort (nested within the combined oral contraceptive cohort), 
women were also required to have a diagnosis of PCOS (ICD-9-CM 256.4). The index date was the first dispensing for a combined oral contraceptive after the PCOS claim. Those with combined oral contraceptive use during the 365-day period before the index date were considered to be prevalent users, while we considered women with no prior combined oral contraceptive use to be new users. We used a logistic regression model to develop a propensity score as the probability for developing PCOS. This score was formed from prescription and medical claims, demographics and health care utilization data during the 365 days before the index date, in addition to the calendar year of diagnosis (formed from covariates at cohort entry in Table 1 and Table 2). This technique is commonly used in large database studies to adjust for a large number of covariates without loss of statistical precision. A 1:1 matching technique, based on the propensity score, was used to select a comparator group of women without PCOS with similar baseline comorbidities (who were also taking combined oral contraceptives).

\section{Outcome measure}

The outcome of nonfatal venous thromboembolism was a combined outcome of pulmonary embolism (ICD-9-CM 414.1) and deep vein thrombosis (ICD-9-CM 453, 451.1). Cases were women who had an event during or within 30 days after cessation of combined oral contraceptive therapy. We included this 30-day window to avoid informative censoring bias, where women may stop taking combined oral contraceptives after having a venous thromboembolism. ${ }^{16}$ All cases were also required to initiate anticoagulant treatment, with the first dose administered within 14 days of the venous thromboembolism claim. The case index date was 14 days after the venous thromboembolism claim to account for the anticoagulant assessment. This approach to identifying venous thromboembolism cases using subsequent anticoagulation therapy has been previously shown to have a $99 \%$ positive predictive value. ${ }^{17}$

\section{Statistical analysis}

In the primary analysis, we used Cox proportional hazards models to estimate the hazard ratio (HR) for venous thromboembolism. We performed a secondary analysis to model the association between PCOS and venous thromboembolism using a modified Poisson regression with a robust error variance, resulting in a risk ratio (RR). ${ }^{18}$

We performed a secondary analysis with a more inclusive definition of PCOS to improve sensitivity and to evaluate the impact of including PCOS diagnostic criteria and treatment in our case ascertainment. Women with a claim for PCOS (ICD-9-CM 256.4), a claim for a diagnostic criteria (anovulation [ICD-9-CM 628.0] and hirsutism [ICD-9-CM 704.1]), or a prescription for antiandrogen treatment (i.e., spironolactone) were included as having PCOS. Spironolactone was included as a proxy for the

Table 2: Comparison of medication use and comorbidities at cohort entry and study end

\begin{tabular}{|llllll}
\hline & \multicolumn{2}{c}{ Cohort entry } & & \multicolumn{2}{c}{ Study end } \\
\cline { 2 - 3 } \cline { 5 - 6 } Variable & PCOS* & $\begin{array}{c}\text { Matched } \\
\text { controls }\end{array}$ & PCOS* & $\begin{array}{c}\text { Matched } \\
\text { controls }\end{array}$ \\
\hline
\end{tabular}

Medication use, \% of women

\begin{tabular}{|c|c|c|c|c|}
\hline ACE inhibitor or ARB & 2.18 & 2.57 & 5.06 & 5.10 \\
\hline Beta blocker & 3.42 & 3.52 & 7.95 & 6.80 \\
\hline Benzodiazepine & 8.93 & 8.06 & 20.77 & 16.95 \\
\hline $\begin{array}{l}\text { Calcium-channel } \\
\text { blocker }\end{array}$ & 0.92 & 1.06 & 2.51 & 2.18 \\
\hline Diabetes medications & 20.31 & 20.65 & 42.88 & 21.55 \\
\hline $\begin{array}{l}\text { SSRI or tricyclic } \\
\text { antidepressant }\end{array}$ & 17.09 & 17.32 & 31.76 & 28.88 \\
\hline
\end{tabular}

$\begin{array}{lllll}\text { Statin or fibrate } & 2.45 & 2.79 & 5.79 & 5.23\end{array}$

Comorbidities, \% of women

$\begin{array}{lllll}\text { Acne } & 11.04 & 10.92 & 24.15 & 19.65\end{array}$

$\begin{array}{lllll}\text { Asthma } & 6.93 & 6.92 & 15.32 & 12.82\end{array}$

$\begin{array}{lllll}\text { COPD } & 3.34 & 3.45 & 11.45 & 9.29\end{array}$

$\begin{array}{lllll}\text { Diabetes } & 6.70 & 8.31 & 14.87 & 12.23\end{array}$

$\begin{array}{lllll}\text { Dysmenorrhea } & 5.79 & 5.71 & 14.23 & 10.95\end{array}$

$\begin{array}{lllll}\text { Endometriosis } & 2.71 & 2.82 & 7.42 & 4.88\end{array}$

$\begin{array}{lllll}\text { Hyperlipidemia } & 10.47 & 10.91 & 27.10 & 20.15\end{array}$

$\begin{array}{lllll}\text { Hypertension } & 7.82 & 8.40 & 18.70 & 14.75\end{array}$

$\begin{array}{lllll}\text { Hypothyroid } & 8.23 & 8.32 & 17.23 & 12.6\end{array}$

$\begin{array}{lllll}\text { Ovarian inflammation } & 1.74 & 1.83 & 5.72 & 3.84\end{array}$

$\begin{array}{lllll}\text { Vaginal inflammation } & 12.56 & 12.52 & 31.22 & 27.64\end{array}$

$\begin{array}{lllll}\text { Uterine leiomyoma } & 2.05 & 2.03 & 5.96 & 4.65\end{array}$

$\begin{array}{lllll}\text { Irregular menstrual } & 40.04 & 40.06 & 72.07 & 54.15\end{array}$
cycle

\begin{tabular}{|lrrrr|}
\hline Migraine & 5.53 & 5.67 & 15.10 & 12.33 \\
\hline $\begin{array}{l}\text { Mood or anxiety } \\
\text { disorder }\end{array}$ & 15.96 & 16.26 & 34.09 & 29.65 \\
\hline Obesity & 13.35 & 13.32 & 33.11 & 21.04 \\
\hline $\begin{array}{l}\text { Peptic ulcer disease } \\
\begin{array}{l}\text { Premenstrual tension } \\
\text { syndrome (PMS or }\end{array}\end{array}$ & 0.40 & 0.42 & 1.32 & 1.03 \\
\hline $\begin{array}{l}\text { PMDD) } \\
\text { Sleep disorder }\end{array}$ & 0.82 & 1.34 & 4.12 & 2.97 \\
\hline Smoking & 2.57 & 2.71 & 7.31 & 6.85 \\
\hline
\end{tabular}

Note: $A C E$ = angiotensin-converting enzyme, $A R B=$ angiotensin-receptor blocker, COPD = chronic obstructive pulmonary disorder, PCOS = polycystic ovary syndrome, $\mathrm{PMDD}=$ premenstrual dysphoric disorder, $\mathrm{PMS}=$ premenstrual syndrome, SSRI = selective serotonin reuptake inhibitor.

*Defined as a claim for PCOS (International Classification of Disease, 9th Revision [Clinical Modification (ICD-9-CM)] 256.4). 
treatment of hyperandrogenism and not as an independent risk factor for venous thromboembolism. As in the main analysis, we used a 1:1 match, based on a propensity score to predict having PCOS to select a comparator population, and we used Cox proportional hazard models to estimate the association between PCOS and venous thromboembolism. All additional PCOS criteria were also evaluated in similar separately matched analyses.

\section{Comparison with women with PCOS not using combined oral contraceptives}

We did not conduct a direct comparison of the risk of venous thromboembolism among women with PCOS using, compared with those not using, combined oral contraceptive therapy; if the decision to treat or not to treat PCOS with combined oral contraceptives is an indication of disease severity, then this analysis would be subject to confounding by indication.

To provide an assessment of baseline venous thromboembolism risk among women with PCOS compared with women without this syndrome, we obtained a random sample of 2 million women not taking combined oral contraceptives. We repeated all analyses as described above, with the exception that a 1:4 match was conducted to ensure adequate power based on the lower prevalence of PCOS among women who do not use combined oral contraceptives.

We evaluated proportionality of hazards using the log-log survival curve.

\section{Results}

We included 1632678 combined oral contraceptive users who met our inclusion criteria. Of these, 46867 women $(2.9 \%$ of the total population) had a claim for PCOS. We were able to successfully match $43506(92.8 \%)$ of these women,

Table 3: Risk of venous thromboembolism associated with PCOS in women taking the combined oral contraceptive pill

\begin{tabular}{|lll|}
\hline Variable & $\begin{array}{c}\text { Cox proportional } \\
\text { hazards models }\end{array}$ & $\begin{array}{c}\text { Relative risk } \\
\text { regression }\end{array}$ \\
\hline PCOS claim & $2.14(1.41-3.24)$ & $2.12(1.40-3.21)$ \\
\hline Composite PCOS definition* & $2.24(1.62-3.10)$ & $2.23(1.61-3.08)$ \\
\hline PCOS symptoms and treatment & & \\
\hline \multicolumn{1}{|c|}{ Anovulation } & $1.72(0.75-3.98)$ & $1.70(0.75-3.89)$ \\
\hline Hirsutism & $2.49(1.35-4.59)$ & $2.43(1.30-4.56)$ \\
\hline Spironolactone & $1.89(1.15-3.10)$ & $1.86(1.14-3.03)$ \\
\hline
\end{tabular}

Note: PCOS = polycystic ovary syndrome.

*Claims for PCOS, anovulation, hirsutism, spironolactone treatment, or PCOS-related procedures. producing a patient population of 87012 for our primary analysis.

We matched women based on comorbidities, assessed during the one year before a claim for PCOS, creating populations with nearly identical characteristics at cohort entry (Table 1). Women with a PCOS claim, however, were more likely to develop nearly every study covariate after baseline, when compared with the matched controls (Table 2). Noteworthy differences at study end include the use of diabetic medications (PCOS: $42.88 \%$ v. controls: $21.55 \%$ ), despite similar rates of diabetes (14.87\% v. $12.23 \%$, respectively), hyperlipidemia $(27.10 \%$ v. $20.15 \%$, respectively), hypertension (18.70\% v. $14.75 \%$, respectively), menstrual irregularity $(72.07 \%$ v. $54.15 \%$, respectively) and obesity $(33.11 \%$ v. $21.04 \%$, respectively).

The incidence of venous thromboembolism among women with PCOS was 23.7/10 000 person-years, while that for matched controls was 10.9/10 000 person-years. Women with PCOS who were taking combined oral contraceptives had an HR for venous thromboembolism of 2.14 (95\% confidence interval [CI] 1.41-3.24). Our secondary analysis found an RR of 2.12 (95\% CI 1.40-3.21) (Table 3).

In our sensitivity analysis, a more inclusive definition of PCOS also included women who met a diagnostic criteria for PCOS (anovulation or hirsutism) or treatment for PCOS (spironolactone). The characteristics of this population are shown in Appendix 1 (available at www.cmaj.ca/lookup /suppl/doi:10.1503/cmaj.120677/-/DC1). This analysis included 89555 women $(5.5 \%$ of the total population) with PCOS, and $84632(94.5 \%)$ were successfully matched, defining a second cohort with 169264 women. In this second analysis, the incidence of venous thromboembolism among women with PCOS was 24.1/10 000 person-years, while that for matched controls was 10.8/10 000 person-years. In this analysis, women with PCOS who were taking combined oral contraceptives had an HR for venous thromboembolism of 2.24 (95\% CI 1.62-3.10) and an RR for venous thromboembolism of 2.23 (95\% CI 1.61-3.08). In the stratified analyses, all additional measures of PCOS in this inclusive definition demonstrated increased risk of venous thromboembolism: anovulation $\mathrm{HR}$ 1.72 (95\% CI 0.75-3.98), hirsutism HR 2.49 (95\% CI 1.35-4.59) and spironolactone HR 1.89 (95\% CI 1.15-3.10).

The incidence of venous thromboembolism was 6.3/10 000 person-years among women with PCOS not taking contraceptives, while the incidence among matched controls was 4.1/10 000 person-years. Among women not taking combined oral contraceptives, women with PCOS 
had an HR for venous thromboembolism of 1.55 (95\% CI 1.10-2.19) and a RR of 1.63 (95\% CI 1.13-2.34) compared with matched controls. The characteristics of this population are shown in Appendix 2 (available at www.cmaj.ca/lookup /suppl/doi:10.1503/cmaj.120677/-/DC1).

\section{Interpretation}

We found a 2-fold increased risk of venous thromboembolism among women with PCOS taking combined oral contraceptives compared with matched controls. We found a similar increased risk when we expanded the definition of PCOS by including its symptoms and treatment. We also found a 1.5 -fold increased relative risk of venous thromboembolism among women with PCOS who were not taking contraceptives compared with matched controls.

Our findings are consistent with the previously reported 2-fold increase in most of the risk factors for venous thromboembolism among women with PCOS. ${ }^{1}$ Among users of combined oral contraceptives, the incidence of venous thromboembolism for matched controls was comparable to that of users in the general population (7-11/10 000 person-years), ${ }^{17,19}$ while the incidence among women with PCOS was doubled. Similarly, among women not using oral contraceptives, the incidence of venous thromboembolism among those without PCOS was close to previously reported baseline rates, ${ }^{17}$ while those with PCOS had increased risk. The absolute rate of venous thromboembolism among women with PCOS taking combined oral contraceptives (23.7/10 000 person-years) was substantially higher than that among women with this condition not taking contraceptives (6.3/10 000 person-years).

Although the covariates at cohort entry were nearly identical between the groups, women with PCOS were more likely to experience nearly every comorbidity during follow-up. Covariates reported at end of the study had a longer period of assessment and thus were better captured; however, the differential development of these conditions supports the finding that women with PCOS are at risk of many additional comorbidities. ${ }^{1}$

The use of contraceptive products by women with PCOS is thought to improve cycle regulation and benefit both acne and hyperandrogenism. ${ }^{20}$ Previous studies have found that combined oral contraceptives are effective for the reduction of androgen levels and hirsutism in women with PCOS; these studies have mainly focused on drospirenone, desogestrel and cyproterone acetate. ${ }^{8,9,19}$ These studies were small in size and not powered to assess thrombotic events.

\section{Limitations}

Because combined oral contraceptives are routinely used by women with PCOS and these drugs are known to increase the risk of venous thromboembolism, including only users of these drugs limits both confounding by indication and contraindication. However, we do not have an accurate measure for many potential confounders (e.g., diet, exercise and family history of venous thromboembolism), and some residual confounding is likely present. Claims for obesity were similar between women with $(13.35 \%)$ and without (13.32\%) PCOS at study entry, suggesting that unmeasured obesity would not be different between groups; however, we did not have access to body mass index values, and residual confounding is possible.

Polycystic ovary syndrome is diagnosed based on the presence of 2 of the following 3 symptoms: oligo- or anovulation, clinical and/or biochemical signs of hyperandrogenism, and polycystic ovaries. ${ }^{1}$ This regimented clinical diagnostic criteria likely makes a claim for PCOS very specific, reducing potential bias from exposure misclassification. However, the sensitivity and specificity of PCOS claims are unknown, and the use of ICD-9-CM codes to identify participants with PCOS may underreport the true prevalence of this condition. Thus, we developed a second definition based on diagnostic criteria and medication treatment in order to perform a second analysis with an improved sensitivity. This inclusive definition gave a prevalence of PCOS of $5.5 \%$ in the study population, which is comparable to the known prevalence of $6 \%-10 \%$, as defined using the National Institute of Health criteria.

\section{Conclusion}

Women with PCOS taking combined oral contraceptives had a 2-fold increased risk of venous thromboembolism, while such women not taking these drugs had a 1.5-fold increased risk. This is consistent with previous finding of increased cardiovascular risk factors and subclinical vascular disease among women with this syndrome. ${ }^{1,5}$ Physicians should be aware of a potentially synergistic increase in venous thromboembolism risk in women with PCOS taking combined oral contraceptives.

\section{References}

1. Fauser BC, Tarlatzis BC, Rebar RW, et al. Consensus on women's health aspects of polycystic ovary syndrome (PCOS): the Amsterdam ESHRE/ASRM-sponsored 3rd PCOS consensus workshop group. Fertil Steril 2012;97:28-38.

2. Rotterdam ESHRE/ASRM-sponsored PCOS consensus workshop group. Revised 2003 consensus on diagnostic criteria and long-term health risks related to polycystic ovary syndrome. Fertil Steril 2004;1:19-25. 
3. Legro RS, Kunselman AR, Dodson WC, et al. Prevalence and predictors of risk for type 2 diabetes mellitus and impaired glucose tolerance in polycystic ovary syndrome: a prospective, controlled study in 254 affected women. J Clin Endocrinol Metab 1999;84:165-9.

4. Ehrmann DA, Barnes RB, Rosenfield RL, et al. Prevalence of impaired glucose tolerance and diabetes in women with polycystic ovary syndrome. Diabetes Care 1999;22:141-6.

5. Moran LJ, Misso ML, Wild RA, et al. Impaired glucose tolerance, type 2 diabetes and metabolic syndrome in polycystic ovary syndrome: a systematic review and meta-analysis. Hum Reprod Update 2010;16:347-63.

6. Baillargeon JP, McClish DK, Essah PA, et al. Association between the current use of low-dose oral contraceptives and cardiovascular arterial disease: a meta-analysis. J Clin Endocrinol Metab 2005; 90:3863-70

7. Kriplani A, Periyasamy AJ, Agarwal N, et al. Effect of oral contraceptive containing ethinyl estradiol combined with drospirenone vs. desogestrel on clinical and biochemical parameters in patients with polycystic ovary syndrome. Contraception 2010;82:139-46.

8. Batukan C, Muderris II. Efficacy of a new oral contraceptive containing drospirenone and ethinyl estradiol in the long-term treatment of hirsutism. Fertil Steril 2006;85:436-40.

9. Falsetti L, Pasinetti E. Effects of long-term administration of an oral contraceptive containing ethinylestradiol and cyprterone acetate on lipid metabolism in women with polycystic ovary syndrome. Acta Obstet Gynecol Scand 1995;74:56-60.

10. Pasquali R, Gambineri A, Anconetani B, et al. The natural history of the metabolic syndrome in young women with polycystic ovary syndrome and the effect of long-term oestrogen-progestagen treatment. Clin Endocrinol (Oxf) 1999;50:517-27.

11. Meyer C, McGrath BP, Teede HJ. Overweight women with polycystic ovary syndrome have evidence of subclinical cardiovascular disease. J Clin Endocrinol Metab 2005;90:5711-6.

12. Bird ST, Pepe SR, Etminan M, et al. The association between drospirenone and hyperkalemia. BMC Clin Pharmacol 2011:11:23.

13. Bird ST, Wei L, Brophy JM, et al. Irritable bowel syndrome and drospirenone-containing oral contraceptives; a comparativesafety study. Curr Drug Saf 2012;7:8-15.

14. Etminan M, Delaney JA, Bressler B, et al. Oral contraceptives and the risk of gallbladder disease: a comparative safety study. CMAJ 2011;183:899-904.

15. Health IMS. LifeLink Health Plan Claims Database: overview and study design issues. Little Rock (AK): University of Arkansas for Medical Sciences; 2010. Available: www.uams.edu /TRI/hsrcore/Lifelink_Health_Plan_Claims_Data_DesignIssues _wcost_April2010[1].pdf (accessed 2012 Nov. 15).

16. Shih W. Problems in dealing with missing data and informative censoring in clinical trials. Curr Control Trials Cardiovasc Med 2002;3:4.

17. Lidegaard $\varnothing$, Nielsen LH, Skovlund CW, et al. Risk of venous thromboembolism from use of oral contraceptives containing different progestogens and oestrogen doses: Danish cohort study, 2001-9. BMJ 2011;343:d6423

18. Zou G. A modified poisson regression approach to prospective studies with binary data. Am J Epidemiol 2004;159:702-6.

19. Seeger JD, Loughlin J, Eng PM, et al. Risk of thromboembolism in women taking ethinyestradiol/drospirenone and other oral contraceptives. Obstet Gynecol 2007;110:587-93.

20. Nader S, Diamanti-Kandarakis E. Polycystic ovary syndrome, oral contraceptives and metabolic issues: new perspectives and a unifying hypothesis. Hum Reprod 2007;22:317-22.

Affiliations: Department of Health and Human Services (Bird), Food and Drug Administration, Center for Drug Evaluation and Research, Office of Management and Academic Collaboration Program; Department of Pharmaceutical Outcome and Policy (Bird, Delaney, Hartzema), University of Florida College of Pharmacy, Gainesville, Fla.; Department of Medicine and Epidemiology (Brophy), McGill University, Montréal, Que.; and the Pharmaceutical Outcomes Programme (Etminan), School of Medicine, University of British Columbia, Vancouver, BC

Contributors: All authors took part in the study design and the analysis and interpretation of the data. The manuscript was drafted by Steven Bird and was critically revised for important intellectual content by all authors. The statistical analysis was completed by Steven Bird, and the study guarantor is Joseph Delaney. All authors approved the final version submitted for publication.

Funding: This work was supported by an unrestricted operating grant funded in part by the McGill University Health Centre, Fonds de la Recherche en Santé du Québec, and the Ministère de la Santé et des Services Sociaux. James Brophy is a physician scientist who receives financial support from le Fonds de la Recherche en Santé du Québec. Joseph Delaney receives financial support from Agency for Healthcare Research and Quality (grant no. R21 HS019516-01). Abraham Hartzema holds a grant from the National Institutes of Health and is the principal investigator for the Observational Medical Outcomes Partnership, a private-public partnership designed to help improve drug safety monitoring.

Acknowledgement: Steven Bird is employed by the Food and Drug Administration. This study represents the opinions of the authors and not those of the Food and Drug Administration. 